\title{
Zur Relevanz der linken Peripherie für die Strukturierung der Information - kontrastive und typologische Überlegungen
}

\begin{abstract}
In der linguistischen Forschung wird häufig auf die besondere informationsstrukturelle Funktion der Randpositionen des Satzes hingewiesen: Diese sind nicht nur für die Gewichtung des satzinternen Materials nach Hintergrund und Vordergrund relevant, sondern tragen auch zur Sicherung der Kohärenz im Diskurs bei. Dabei wird vor allem mit Hinweis auf kognitive und funktionale Prinzipien die universelle Gültigkeit der Topik-Fokus-Abfolge betont, mit der initialen (oder frühen) Platzierung des kontextuell verankerten Satzgegenstandes (des so genannten Topiks) und der späteren Erwähnung der Kernaussage (des Fokus).

In dem vorliegenden Artikel wird die Relevanz des linken Satzrandes für die Herstellung des Diskurszusammenhanges und die Universalität der Topik-FokusOrdnung überprüft. Zum einen scheint die Funktion des Satzanfangs komplex: Mit dem ersten Glied der Aussagesätze wird nämlich in vielen Sprachen nicht nur an etwas Gesagtes angeschlossen, sondern kann auch etwas Wichtiges hervorgehoben werden. Zum anderen sind die Unterschiede zwischen den Sprachen beträchtlich, was auf den Einfluss struktureller Gegebenheiten hindeutet.

Zur Lösung der Probleme wird ein neues informationsstrukturelles Prinzip vorgeschlagen, das auf der „C-Markiertheit“ (Kohärenzmarkiertheit) der Konstituenten aufbaut. Demnach sind nicht nur kontextuell gegebene, sondern auch kontrastive Elemente relevant für die Herstellung der Kohärenz (und somit C-markiert), da diese die Zuordnung zu ähnlichen oder identischen Mengen bzw. Skalen voraussetzen. Als universelle Strategie der pragmatischen Ordnung ist somit der so genannte „C-Constraint“" zu betrachten, der die initiale Platzierung der C-markierten Konstituenten vorschreibt, unabhängig von ihrem Topik- oder Fokusstatus.

Der typologischen Vielfalt bezüglich der Besetzung des linken Satzrandes wird durch die Beachtung relevanter struktureller Parameter in sieben genetisch und typologisch unterschiedlichen europäischen Sprachen (Deutsch, Englisch, Schwedisch, Französisch, Russisch, Finnisch, Ungarisch) - und durch die Aufstellung der so genannten „C-Hierarchie“ - Rechnung getragen. Deren drei Hauptkategorien weisen bezüglich der Zulassung von C-markierten kontrastiven Elementen und von unterschiedlichen Typen nicht C-markierter Fokusglieder am Satzanfang eine bedeutende Variation auf.
\end{abstract}

\section{Einleitung}

Beim Sprachvergleich richtet sich oft die Aufmerksamkeit auf die kontrastive Analyse struktureller Besonderheiten der Sprachen. Im vorliegenden Artikel werden allerdings über die Beachtung von relevanten grammatischen Gegebenheiten hinaus die Möglichkeiten verschiedener Sprachen zur Siche- 
rung der adäquaten Einbettung sprachlicher Strukturen in den größeren sprachlichen und situativen Kontext in Betracht gezogen. Bei der kontextuellen Einbettung bzw. bei der Sicherung des textuellen Zusammenhanges sind die Randpositionen des Satzes - vor allem der Satzanfang - besonders relevant. In einigen Sprachen, z.B. im Englischen, ist die Füllung der satzinitialen Position grammatisch festgelegt: Im unmarkierten Falle - in Aussagesätzen - steht das Subjekt am Anfang des Satzes. In vielen Sprachen aber - so auch im Deutschen - eröffnet sich ein Spielraum für die Anpassung des Satzes an den Kontext durch die Wahl der satzinitialen Konstituente.

Die Füllung der satzinitialen Position ist im Deutschen relativ frei: Wie die aus Thomas Manns Roman „Die Bekenntnisse des Hochstaplers Felix Krull“" zitierten Beispiele auch zeigen, können Argumente, Adverbiale und auch infinite Prädikatsteile am Satzanfang erscheinen. In neutralen Fällen wie bei der Aussage in Beispiel (1) leitet das Subjekt den Satz ein, aber die satzinitiale Stellung des Objekts des Satzes ist auch möglich (vgl. Beispiel (2)):

(1) Mein armer Vater war Inhaber der Firma Engelbert Krull ... (S. 9)

(2) Kontrakte kann man brechen. (S. 259)

Auch verschiedene Adverbiale und Prädikatsteile können satzeinleitend auftreten:

(3) Beim Sonntagsdiner wurde viel Champagner getrunken im Saal. (S. 232)

(4) Oft hörte ich aus dem Munde der Meinen, daß ich ein Sonntagskind sei. (S. 13)

(5) In der Tat grenzte meine Begabung zur Liebeslust ans Wunderbare; ... (S. 55)

(6) Scblecht verköstigt und schlecht beherbergt war ich in diesem der Zahlkraft üppig sich anbietenden Hause ... (S. 199)

(7) Gut Kirschenessen ist allerdings nicht mit der. (S. 311)

Wie in der einschlägigen Literatur häufig hervorgehoben wird, ist die linke Satzperipherie besonders relevant für die Sicherung der Kohärenz. Es ist aber auch eine alte Einsicht der Forschung, dass die Funktion der linken Randposition im deutschen Aussagesatz komplex ist: Laut Duden (1998) kann diese Position den Anschluss sichern - und dadurch die Realisierung der Topik-vor-Fokus-Anordnung, d.h. die Nachstellung der neuen wichtigen Elemente - ermöglichen, wie in Beispiel (8):

(8) Peter war gestern im Schwimmbad. Er war drei Stunden dort. Dann ging er ins Kino. 
Die Erstposition kann aber auch eine so genannte „Ausdrucksstellung“ sein, die dem Ausdruck von Emphase oder der Andeutung eines Gegensatzes dient:

\section{(9) Niemals wird das geschehen!}

Eine adäquate Beschreibung und Erklärung der Variation bei der Füllung des linken Satzrandes verlangt somit die Bezugnahme auf die Interaktion zwischen syntaktischen Regularitäten und den pragmatischen Möglichkeiten bzw. Restriktionen, d.h. einen so genannten „Interface“-Ansatz. Gleichzeitig setzt die kontrastive Orientierung die Erforschung von informationsstrukturellen Universalien und die Beachtung der sprachspezifischen Variation voraus.

Im Folgenden möchte ich mich auf die „fundamentalen“ Aspekte der Informationsstruktur bezüglich des Satzanfangs in Aussagesätzen konzentrieren, der in der deutschen Fachliteratur bezüglich des Deutschen als „Vorfeld“, in der schwedischen Akademiegrammatik als „Fundament" bezeichnet wird. Dabei werde ich einige grundlegende theoretische Begriffe und das Inventar informationsstruktureller Konzepte erörtern, die für die Erklärung der Funktion(en) des Vorfelds notwendig sind. Abschließend gehe ich auf der Grundlage von sprachlichen Universalien und mit Bezugnahme auf die sprachspezifische Variation auf die Diskussion von möglichen Lösungen ein.

Die Probleme bei der Besetzung der satzinitialen Position, die ich ansprechen möchte, sind die folgenden:

(i) Neben der allgemeinen Tendenz, das Topik vor dem Fokus zu platzieren, gibt es auch die Möglichkeit, die Anfangsposition als „Ausdrucksstellung" zu realisieren.

(ii) Genetisch und typologisch verwandte Sprachen - wie das Deutsche und das Schwedische - weisen dabei Unterschiede auf: Während im Deutschen die Variation im Vorfeld größer ist als im Schwedischen und hier oft auch gewichtige Satzglieder vorkommen, ist im schwedischen Fundament das Subjekt (als Nominalphrase oder Pronomen realisiert) oder ein pronominales Objekt die frequenteste (intonatorisch nicht notwendigerweise markierte) Option.

(iii) Gleichzeitig kann man in genetisch und typologisch unterschiedlichen Sprachen wie im Französischen und Schwedischen ähnliche Tendenzen beobachten: Sowohl das Schwedische als auch das Französische - und dies gilt vor allem für die französische Standardsprache - zeigen eine starke Präferenz für die Stellung intonatorisch nicht markierter Elemente am Satzanfang. 
Bezüglich der strukturellen und funktionalen Charakteristik der linken Satzperipherie stehen folgende Fragestellungen im Zentrum des Interesses:

(i) Wie kann der Satzanfang die Kohärenz im Text auf eine optimale Weise sichern?

(ii) Ist die Topik-Fokus-Abfolge universell? Ist die satzinitiale Platzierung des Topiks in allen Sprachen optimal?

(iii) Wie lässt sich die beobachtete sprachspezifische Variation bezüglich des Satzanfangs erklären? Worin liegen dabei die Unterschiede zwischen dem Deutschen und Schwedischen bzw. die Ähnlichkeiten zwischen dem Schwedischen und Französischen?

(iv) Ist die Variation durch strukturelle oder funktionale Faktoren bedingt?

Die Diskussion der erwähnten Fragen verlangt auch eine Problematisierung des informationsstrukturellen Begriffsinventars: In der Forschung werden häufig die Begriffe Topik und Fokus als die wichtigsten Begriffe der Informationsstruktur (IS) betrachtet, da diese Größen der zweifachen Anforderung der Informationsstrukturierung entsprechen: (i) der Sicherung der Kohärenz (Topik) und (ii) der Sicherung der Informativität (Fokus). Es erhebt sich aber die Frage, ob diese Begriffsdichotomie für die Analyse der Funktion der satzinitialen Position ausreicht oder das Begriffsinventar zu erweitern ist.

Die vorgeschlagenen Lösungen setzen meines Erachtens auch eine kritische Überprüfung der bisher vorgeschlagenen universellen Prinzipien der Informationsstrukturierung (IS) voraus. In dem vorliegenden Artikel wird ein neues Prinzip der Informationsstrukturierung vorgeschlagen, das als „Kohärenzprinzip“ bezeichnet wird. Mit Berücksichtigung des Zusammenhanges zwischen verschiedenen Topik- und Fokustypen und der Kohärenzmarkierung wird das Begriffsinventar der IS erweitert und eine neue Abstraktionsebene der IS-Begriffe eingeführt. Ich werde auch nachzuweisen versuchen, dass das pragmatische Potenzial der satzinitialen Position außer von kognitiven und diskurspragmatischen auch von strukturellen und prosodischen Faktoren gesteuert bzw. eingeschränkt wird, was die Beachtung der sprachspezifischen Variation verlangt.

Der Artikel ist demnach wie folgt aufgebaut: Im nächsten Abschnitt (Abschnitt 2) werden relevante universelle Prinzipien der Informationsstrukturierung zusammengestellt und einige mit ihnen verbundene Probleme und Widersprüche angesprochen. In Abschnitt 3 wird das Kohärenzprinzip dargestellt und in Abschnitt 4 erfolgt die Revision des Begriffsinventars der Informationsstrukturierung auf der Grundlage des Begriffs Kohärenzmarkierung. Daraufhin wird in Abschnitt 5 auf die typologische Variation bezüglich des linken Satzrandes eingegangen, und es werden die verschiedenen Sprachgruppen in einer Hierarchie präsentiert. Abschnitt 6 enthält 
Überlegungen zum Deutschen im Sprachvergleich, mit Fokussierung auf den Unterschied zwischen dem Deutschen und dem Schwedischen. Abschließend (in Abschnitt 7) werden die Schwerpunkte des Artikels zusammengefasst.

\section{Die relevanten Prinzipien der Informationsstrukturierung}

In der Forschung gibt es eine Reihe von Vorschlägen bezüglich der Frage, welche Prinzipien bzw. Tendenzen für die Informationsstrukturierung als universell gültig betrachtet werden können. Bei den meisten Vorschlägen wird auf die Relevanz der kognitiven Faktoren und der kommunikativen Situation hingewiesen, in einigen Fällen wird aber auch auf strukturell bedingte Regularitäten zurückgegriffen. Als erstes Prinzip in diesem Zusammenhang verdient das von Gundel (1988, S. 229) vorgeschlagene Given Before New Principle Erwähnung: „State what is given before what is new in relation to it." Seit der Prager Schule wird gerade dieses Prinzip - oder die so genannte „Thema-Rhema-Abfolge“ - als grundlegend für die Informationsstrukturierung betrachtet. Dieses auf kognitiven und kommunikativen Strategien aufbauende Prinzip ist mit anderen bekannten Prinzipien der Forschung eng verwandt, so mit dem Theme First Principle (vgl. Tomlin 1986, S. 37), laut dem ,[...] information that is more ,thematic tends to precede information that is less ,thematic" " und mit dem von Hetzron (1975) eingeführten und nach Herring (1990, S. 164) zitierten Discourse Iconicity Principle, das besagt: „Information ideally is placed as close as possible to the part of the discourse to which it relates; i.e. sentences start with what has already been talked about, and end with what is to be talked about next.“

Ein weiteres funktionales Prinzip steht aber im Gegensatz zu den bisher erwähnten Prinzipien. Laut diesem Prinzip, das bei Gundel (1988, S. 229) als First Things First Principle bezeichnet wird, enthält der Satzanfang den Informationskern: „Provide the most important information first.“ Dieses Prinzip erscheint ebenfalls in mehreren Varianten in der Literatur, bei Mithun (1987, S. 304) als Newsworthiness Principle und auch bei Givón (1988), der für die universelle Geltung dieses Prinzips argumentiert, mit der Formulierung ,Attend first to the most urgent task“.

Über die bisher erwähnten funktionalen und kognitiven Prinzipien hinaus wird aber in der Forschung auch oft auf die Relevanz von intonatorischen bzw. rhythmischen Faktoren und strukturellen Aspekten hingewiesen. Das Gewicht der Satzglieder als relevanter Steuerfaktor wird schon bei Beauzée (1767) beachtet, dieses Prinzip ist aber vor allem seit Behaghels (1932) Vorschlag als Gesetz der wachsenden Glieder bekannt. In neueren Arbeiten wird auch der Einfluss des Rhythmus - der rhythmischen Alternation 
und Wohlgeformtheit - auf die Informationsstrukturierung hervorgehoben, vor allem in dem von Monika Doherty (2003) vorgeschlagenen BID-Prinzip, dem Balanced Information Distribution Principle.

Die Relevanz der strukturellen Faktoren wird vor allem in typologisch orientierten Arbeiten angesprochen. In diesem Zusammenhang ist vor allem Herrings (1990, S. 164) Word Order Type Principle (WOP) zu erwähnen, laut dem , ,...] information structure is determined relative to a language's basic word order" (siehe unten die Diskussion der Relation zwischen der Basisgliedfolge zur Platzierung von Topik und Fokus in (10)).

Im Zusammenhang mit den universellen Prinzipien ist es allerdings notwendig, auf einige Probleme und Widersprüche hinzuweisen. Eines der Probleme besteht darin, dass die auf kognitive und funktionale Strategien rekurrierenden so genannten „Natürlichkeitsprinzipien“ („naturalness principles“) widersprüchlich sind. Wie wir oben sehen konnten, wird in der Forschung nicht nur die Natürlichkeit der „Topik-vor-Fokus“-Abfolge behauptet, sondern auch die des entgegengesetzen Prinzips „Fokus-vor-Topik“. Der Anspruch auf die Universalität des erstgenannten Prinzips (Herring 1990, S. 163) "Constituents which relate thematically to the preceding discourse come first, while referents with new (,focused') information appear later, closer to the subsequent discourse in which they play a part" widerspricht der besonders von Givón (1988) befürworteten These, laut der ,the general state of affairs for language is that non-urgent, i.e. predictable or less important, information tends to come late and urgent, i.e. unpredictable or important, information early". Somit ist die Frage, welchem Prinzip in der Informationsstrukturierung universelle Gültigkeit zuzuschreiben ist, in der Forschung bisher noch nicht zufriedenstellend beantwortet worden.

In denjenigen Fällen, wo bei der Diskussion der universellen Prinzipien auch typologische Unterschiede beachtet werden, richtet sich die Aufmerksamkeit vor allem auf die Basisgliedfolge. Die zwei typologisch relevanten Klassen in diesem Zusammenhang bilden Sprachen, in denen in unmarkierten Fällen die Aussagesätze verbinitial sind (die so genannten VS-Sprachen) und Sprachen, in denen in diesen Fällen das Subjekt vor dem Verb steht (die SV-Sprachen). Herring (1990) behauptet, dass die zuverlässigste Voraussage bezüglich der Anordnung von Topik und Fokus auf der Grundlage des WOP gemacht werden kann: In verbinitialen Sprachen steht der Fokus am Satzanfang, während nicht-verbinitiale Sprachen am Satzanfang das Topik bevorzugen: ,[...] the word order type principle predicts that topics of either type will be preposed in SV languages but postposed in VS languages, while foci will be postposed in SV languages but preposed in VS languages" (ebd., S. 166). Dies wird in (10) illustriert: 
(10)

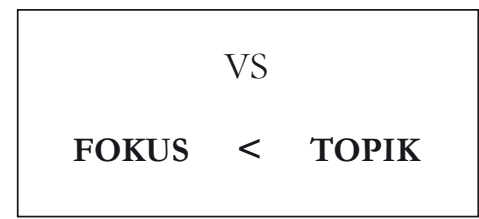

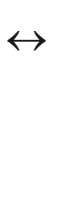

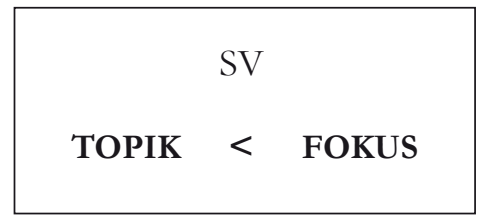

Herring hebt allerdings hervor, dass keine vollständige Korrelation zwischen dem WOP und der Topik-Fokus-Abfolge herrscht: In bestimmten Fällen können nämlich die kognitiv und funktional verankerten Natürlichkeitsprinzipien das strukturelle Prinzip - das WOP - überspielen. Wie in (11) gezeigt wird, scheinen beide Sprachtypen bezüglich der Platzierung der kontrastiven Elemente, die unabhängig von der Basisgliedfolge in beiden Sprachtypen satzinitial stehen, konsequent zu sein:

(11)

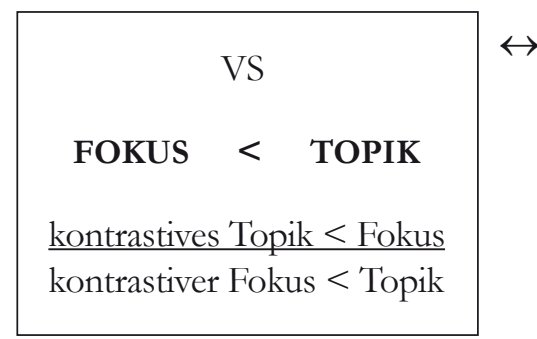

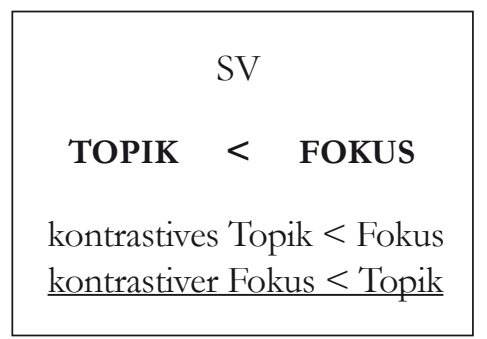

Das kontrastive Topik (so genannte „Shifted Topic") ist somit am Satzanfang nicht nur in SV-Sprachen, sondern auch in VS-Sprachen möglich, in diesem letzteren Fall im Widerspruch zum WOP (die diesem Prinzip widersprechende Abfolge ist oben durch Unterstreichung markiert). Wie wir zudem sehen können, liegt auch in SV-Sprachen eine Spannung zwischen dem strukturell verankerten WOP und den Natürlichkeitsprinzipien vor, in diesem Fall bezüglich der Platzierung des kontrastiven Fokus, der nach vorne bewegt werden kann. Weiter unten wird anhand von konkreten sprachlichen Beispielen aus einigen SV-Sprachen gezeigt, dass für die Auflösung dieser Spannung die Satzspaltung (vor allem ,it-clefts') besonders gut geeignet ist. Diese Konstruktion ermöglicht nämlich gleichzeitig die Voranstellung und Nachstellung des kontrastiv fokussierten Elements: Dies wird vom Rest des Satzes getrennt und in einem Matrixsatz links von dem untergeordneten Satz realisiert, steht aber häufig weiter rechts im Matrixsatz, der mit einem „leichten“, expletiven Pronomen (it, es) eingeleitet wird. ${ }^{1}$

Es ist allerdings wichtig zu beachten, dass die Funktion der Satzspaltung (bei it-clefts) nicht auf die Markierung des im Matrixsatz stehenden kontrastiven Fokus beschränkt ist. Vgl. hierzu Prince (1978), Declerck (1984) und Huber (2002, 2006). 


\section{Das Kohärenzprinzip}

Die bisher erwähnten Probleme und typologischen Beobachtungen lassen darauf schließen, dass weder die „Topik-Fokus-Abfolge“ noch die „FokusTopik-Abfolge" als eine universelle Gesetzmäßigkeit betrachtet werden kann. Für die Lösung der Probleme und der Widersprüche wird somit ein neues Prinzip vorgeschlagen - das so genannte Kohärenzprinzip (vgl. Molnár 1993). Dieses Prinzip ermöglicht eine Erklärung, warum kontrastive Topiks und kontrastive Foki dem WOP widersprechend nach vorne bewegt werden: Aufgrund des Kohärenzprinzips („C-Constraint") ist es nämlich notwendig, den Satz mit einem Satzglied einzuleiten, das den textuellen Zusammenhang sichert. Dies ist entweder aufgrund der kontextuellen Gegebenheit bzw. Bekanntheit des initialen Elementes möglich oder aufgrund des Kontrasts. Das Kohärenzprinzip besagt demnach, dass für die Platzierung der Elemente nicht der Topik- oder Fokus-Status entscheidend ist, sondern ihr Beitrag zur Kohärenzsicherung.

Wie in (12) gezeigt, stehen C-markierte (kohärenzsichernde) Elemente - Topiks oder Foki - vor nicht-kohärenzsichernden Elementen, die den negativen Wert des C-Merkmals tragen (vgl. Molnár/Winkler 2009):

$$
\begin{aligned}
& \text { Universelle informationsstrukturelle Strategie: Kohärenzprinzip } \\
& \text { („C-Constraint“") }
\end{aligned}
$$

$$
\begin{aligned}
& \text {,[+C]-markiert“ } \quad<\quad,[-\mathrm{C}] \text {-markiert" } \\
& \text { (Topik oder Fokus) (Topik oder Fokus) }
\end{aligned}
$$

Den Beitrag der initial platzierten Konstituenten zur Kohärenz möchte ich im Folgenden mit Rückgriff auf das C-Merkmal - das Kohärenzmerkmal - diskutieren. Auch wenn ich das C-Merkmal dem Vorschlag der Generativen Grammatik - Chomskys (1995) „,Feature-based approach“ (vgl. auch Rizzi 1997; Jackendoff 2002) - folgend als ein syntaktisches Merkmal betrachte, liegt diesem Merkmal eine pragmatische Eigenschaft zugrunde, die erst aufgrund von kontextuellen Gegebenheiten spezifizierbar ist. Wie in (13) gezeigt, kann das Kohärenzmerkmal entweder mit einem positiven oder negativen Wert auftreten. Das positive C-Merkmal hat allerdings zwei Varianten: „C-Kontinuität“ und „C-Kontrast“. C-Kontinuität setzt die Identität oder Ähnlichkeit der Entitäten voraus, während C-Kontrast dies ausschließt. C-Kontrast verlangt jedoch die Zugehörigkeit der Elemente zu einer identischen Menge oder Skala. Schließlich ist der negative Wert des C-Merkmals auch denkbar in den Fällen, wo weder Identität, Ähnlichkeit der Elemente selbst noch deren Zugehörigkeit zu einer identischen Menge oder Skala vorliegt. 
(13) „C-Merkmal“ - Differenzierungen:

$$
\begin{array}{cl}
{[+\mathrm{C}]-[+\mathrm{C}]-\text { Kontinuität: }} & \mathrm{X} i=\mathrm{X} j \\
-[+\mathrm{C}]-\text { Kontrast: } & \mathrm{X} i \neq \mathrm{X} j \\
& \text { aber Zugehörigkeit zu einer identischen } \\
& \text { Menge oder Skala } \\
{[-\mathrm{C}]-\mathrm{X} i \neq \mathrm{X} j} & \text { keine Zugehörigkeit zu einer identi- } \\
& \text { schen Menge oder Skala }
\end{array}
$$

Für die linke Peripherie sind vor allem die Elemente von Interesse, die entweder den positiven Wert für die C-Kontinuität oder für den C-Kontrast -

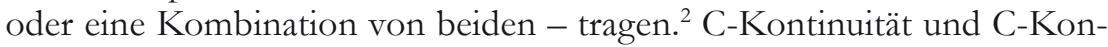
trastivität können aber nicht als homogene Begriffe betrachtet werden, und ihrem Beitrag zur Kohärenzetablierung kann nur dann Rechnung getragen werden, wenn sie weiter differenziert werden.

Zur Differenzierung der C-Kontinutät - bzw. der damit verwandten Begriffe - liegen in der Forschung zahlreiche Vorschläge vor, unter anderem in den Arbeiten von Chafe $(1974,1976)$, Prince $(1981,1985)$ und Gundel et al. (1993). Gundel et al. (1993) bezeichnen den stärksten Grad von "Givennes" als „,in focus“, den schwächsten Grad als „type identifiable“ und unterscheiden vier weitere Typen auf der Hierarchie: ,activated“, „familiar", „, uniquely identifiable“ und ,referential“. Das Diagramm in (14) zeigt auch, dass der erste Typ der „Givenness Hierarchy“ - nämlich der Typ, der als , in focus" bezeichnet wird (gemeint: ,in focus of interest", p.c. J. Gundel) - der wichtigste Typ für die [+]-C-Markierung ist und auch im größten Maße mit dem Topik zu korrelieren scheint (die für Kohärenzetablierung relevanten Typen sind in (14) fettgedruckt):

(14) „The Givenness Hierarchy“ (Gundel et al. 1993)

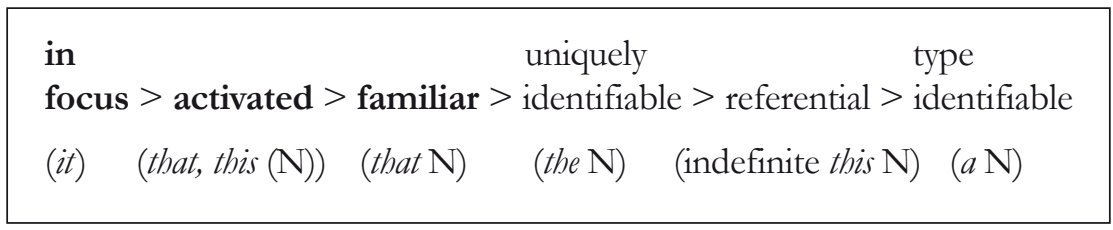

TOPIK FOKUS $[+\mathrm{C}]$

$2 \quad$ Kontrastive Elemente sind häufig bereits erwähnt und kontextuell verankert, vgl. folgendes Beispiel:

(i) A: Wir möchten im Sommer entweder nach Spanien oder nach Island fahren, haben uns aber noch nicht entscheiden können.

B: Von Spanien würde ich euch abraten, es ist dort im Sommer zu heiß. 
Allerdings ist nicht nur beim Begriff der „Kontinuität“, sondern auch beim „Kontrast-Begriff“" eine weitere Differenzierung vonnöten (vgl. unten die „Contrast Hierarchy“ in (15)). In früheren Arbeiten versuchte ich zu zeigen, dass für die Definition des Kontrastbegriffs in der Forschung unterschiedliche Kriterien verwendet werden (vgl. Molnár 2002, 2006; Molnár/Winkler 2009). In verschiedenen Arbeiten wird aber betont, dass prototypischer Kontrast nur dann vorliegen kann, wenn die Alternativen kontextuell restringiert sind und explizit erwähnt werden. In diesen Fällen ist der Kontrast „pragmatisch fundiert“. Diesen Kontrasttypen kann somit das positive CMerkmal zugewiesen werden (die für die C-Markierung relevanten Typen werden auch in (15) fettgedruckt). Bei den anderen Kontrastdefinitionen ist allerdings das Vorliegen bzw. die kontextuelle Verankerung der Alternativen nicht unbedingt notwendig. In diesen Fällen ist der Kontrastbegriff entweder mit der Definition des Fokus deckungsgleich (indem Kontrast nur als „highlighting“ definiert wird) oder er entspricht der Definition von ,narrow focus“ (dieser Typ wird unten mit Dretske 1972 als „dominant contrast“" bezeichnet). Die Einschränkung der Fokusdomäne führt zwar häufig zur Herstellung eines Bezugs zu Alternativen, in einigen Sprachen sind allerdings weitere prosodische und syntaktische Mittel notwendig für die „Alternativenbezug“"-Lesart von „,narrow focus“. 3

Im Ungarischen kann der enge Fokus sowohl postverbal als auch präverbal stehen, wobei nur die präverbale Platzierung der fokussierten Konstituente Alternativenbezug andeutet.

Vgl. Molnár (2006, S. 201 f.): „Along the lines of É.Kiss's argumentation, in a sentence like $\left[I-\mathrm{B}_{1}\right]$ as an answer to the question Where have terror attacks taken place? the postverbal constituent Madrid merely presents non-presupposed information without suggesting that the focussed constituent is the only one of the relevant entities predicated on (unfortunately corresponding to the actual political reality), whereas in [II- $\left.\mathrm{B}_{2}\right]$ the same constituent standing in the preverbal focus position exhaustively identifies the relevant set of places among those present in the domain of discourse:

$$
\begin{aligned}
& \text { A: Where have terror attacks taken place? } \\
& \mathrm{B}_{1} \text { : Terrortámadás volt MADRIDBAN. } \\
& \text { terror attack was Madrid-in } \\
& \text {,Terror attacks have taken place in Madrid.“ }
\end{aligned}
$$

$$
\begin{aligned}
& \mathrm{B}_{2}: \text { MADRIDBAN volt terrortámadás. } \\
& \text { Madrid-in was terror attack }
\end{aligned}
$$

,It was in Madrid that terror attacks have taken place.““

${ }_{4} \quad$ In Antworten auf Konstituentenfragen sind die Alternativen nicht unbedingt kontextuell verankert:

(iii) A: Wo möchtest Du im Sommer Deinen Urlaub verbringen?

B: In Spanien.

Die relevanten Alternativen können aber auch im Kontext spezifiziert sein:

(iv) A: Wo möchtest Du im Sommer Deinen Urlaub verbringen, in Eurer Hütte oder in Spanien?

B: In Spanien. 
(15) „The Contrast Hierarchy“ (vgl. Molnár 2006)
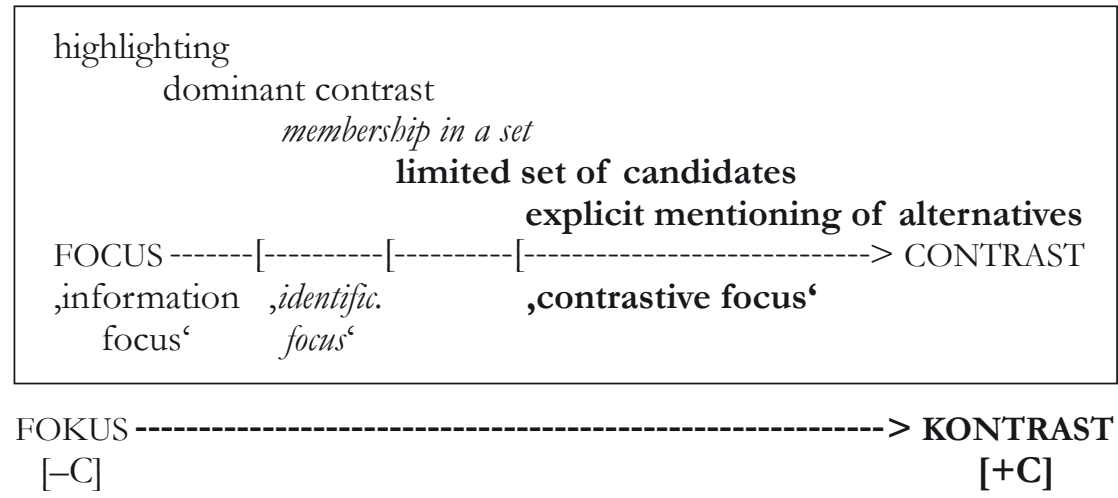

Alternativenbezug ist jedoch relevant für den im mittleren Teil der Hierarchie stehenden Typ, der semantisch - aufgrund des Kriteriums „set membership “ - erfasst wird. Die kontextuelle Verankerung des Kontrasts ist aber auch hier nicht zwingend. ${ }^{4}$ Dieser Kontrasttyp (der dem von É.Kiss (1998) vorgeschlagenen „Identificational Focus “5 entspricht) wurde von Vallduví/ Vilkuna (1998, S. 83-84) folgendermaßen definiert:

The basic idea behind the notion of kontrast is the following: if an expression $a$ is kontrastive, a membership set $\mathrm{M}=\{\ldots, \mathrm{a}, \ldots\}$ is generated and becomes available to semantic computation as some sort of quantificational domain [...]

Für die [+]-C-Markierung sind allerdings nur diejenigen Typen des Kontrasts von Relevanz, bei denen die Menge der Alternativen vom Kontext eingeschränkt wird bzw. die Gegenalternativen aus dem Kontext deutlich hervorgehen (siehe Fußnote 4).

\section{Zum Begriffsinventar der Informationsstrukturierung}

Wie zuvor schon anhand des Kohärenzprinzips betont wurde, ist nicht der Topik- bzw. Fokus-Status der Konstituenten für ihre Platzierung am Satzanfang entscheidend, sondern ihr Status bezüglich der C-Markiertheit. CMarkiertheit motiviert demnach die Einführung einer zusätzlichen Abstrak-

Zur Definition von „Identificational Focus“ vgl. É.Kiss (1998, S. 245): „An identificational focus represents a subset of the set of contextually or situationally given elements for which the predicate phrase can potentially hold; it is identified as the exhaustive subset of this set for which the predicate phrase actually holds."

„Identificational Focus“ wird dem anderen Fokustyp - „Information Focus“ - gegenübergestellt. Vgl. É.Kiss (1998, S. 246): „If a sentence part conveys new, nonpresupposed information marked by one or more pitch accents - without expressing exhaustive identification performed on a set of contextually or situationally given entities, it is not an identificational focus but a mere information focus." 
tionsebene ins Begriffsinventar der Informationsstruktur. Das komplexe Zusammenspiel zwischen Topik, Fokus und C-Markiertheit wird in Tabelle (16) zusammengefasst:

(16) IS-Begriffe TOPIK, FOKUS, KONTRAST - auf der Grundlage der „C-Markierung“"

\begin{tabular}{|c|c|c|}
\hline [+ C-Kontinuität] & [+C-Kontrast $] \quad$ vs. & {$[-\mathrm{C}]$} \\
\hline „continuous TOPIC“ & $\begin{array}{l}\text { contrastive TOPIC } \\
\text { contrastive FOCUS } \\
\text { CONTRAST } \\
\text { (Topic/Focus) }\end{array}$ & $\begin{array}{l}\text { new TOPIC } \\
\text { information FOCUS }\end{array}$ \\
\hline
\end{tabular}

Wie die Tabelle oben zeigt, ist der positive Wert von C-Kontinuität für „continuous (given) topics“ charakteristisch, [+]-C-Kontrast liegt bei kontrastiven Topiks und Foki vor, während das C-Merkmal bei neuen Topiks und bei dem Informationsfokus einen negativen Wert trägt. Ganz wesentlich für unseren Zusammenhang ist demnach, dass beide Begriffe - Topik und Fokus - mit oder ohne C-Markierung möglich sind und dass nur die [+]-C-markierten Topik- und Fokustypen für die Etablierung der Kohärenz geeignet sind, die [-]-C-markierten Kategorien dagegen - wie neue Topiks und nicht-kontrastive Foki - nicht.

\section{Die „C-Hierarchie“}

Nach der Diskussion relevanter Prinzipien und Begriffe der Informationsstrukturierung werden im Folgenden sieben europäische Sprachen näher untersucht - im Hinblick auf die Frage, auf welche Weise die linke Peripherie in diesen Sprachen zur Sicherung der Kohärenz beiträgt. Diese Sprachen gehören alle zu der Kategorie, die bei Herring (1990) als SV-Sprachen, d.h. nicht-verbinitiale Sprachen figurieren. Wie bereits erwähnt, ist für diese Sprachen in Herrings Modell die Topik-Fokus-Abfolge kennzeichnend. In dem hier vorgeschlagenen Rahmen bedeutet dies, dass die C-Markierung am Satzanfang sowohl in der C-Kontinuitäts-Variante - bei den prototypischen Topiks - relevant ist als auch beim Vorliegen eines Kontrasts.

Wie aber in den unten stehenden Überlegungen gezeigt wird, verhalten sich die sieben untersuchten - genetisch und typologisch teilweise unterschiedlichen - Sprachen nicht einheitlich. Während in einigen von ihnen - in der ersten Kategorie - [+]-C-Kontinuität eine starke Dominanz aufweist, ist [+]-C-Kontinuität zwar auch für die zweite Kategorie relevant, bei dieser Kategorie ist allerdings der [+]-C-Kontrast auch frequent (sogar gram- 
matikalisiert). Die dritte Kategorie ist trotz der Relevanz des positiven Werts der C-Markierung in höherem Grade flexibel bezüglich der [-]-C-markierten Optionen am Satzanfang, nämlich bezüglich des kontextuell nicht verankerten, neuen Topiks und des Informationsfokus.

In der unten in (17) aufgestellten „C-Hierarchie ${ }^{\text {“6 }}$ werden die sieben europäischen Sprachen den einzelnen Kategorien folgendermaßen zugeordnet: Zu der ersten Kategorie gehören das Französische und das Schwedische, zu der zweiten das Finnische und das Russische und zu der letzten Kategorie das Englische, Deutsche und das Ungarische. Wie aus der C-Hierarchie ersichtlich, ist die genetische Verwandtschaft der Sprachen nicht entscheidend. Die Relevanz der typologischen Charakteristika wird aber bei der Diskussion der verschiedenen Typen später unten in den Abschnitten 5.1, 5.2 und 5.3 angesprochen. Bereits an dieser Stelle ist es aber wichtig, darauf hinzuweisen, dass zwei typologisch und genetisch besonders eng verwandte Sprachen der Hierarchie - das Deutsche und das Schwedische - in unterschiedliche Kategorien eingeordnet werden.

\section{(17) Die „C-Hierarchie“}

\begin{tabular}{|c|c|c|c|}
\hline$[+\mathrm{C}]$ & $<$ & {$[+\mathrm{C}]$} & {$[-\mathrm{C}]$} \\
\hline „C-Kontinuität" & $<$ & „C-Kontrast" & \\
\hline
\end{tabular}

Bei der typologischen Charakteristik der Sprachen bilden in der Forschung verschiedene strukturelle Eigenschaften und Parameter die Grundlage. Diese Parameter werden mit Rückgriff auf die Morphologie, Phonologie und Syntax aufgestellt. Bezüglich der Morphologie sind die Differenziertheit des Pronomensystems, das Artikelsystem und die Kasusmorphologie bedeutend. Bezüglich der Phonologie ist vor allem die Direktionalität der „Nuclear Stress Rule" (NSR; Chomsky/Halle 1968) zu erwähnen, aber auch der Unterschied zwischen „plastic“" vs. „,non-plastic“ intonation (Vallduví 1992, siehe Abschnitt 5.1) und das Vorliegen distinkter Akzentmuster wie (,fall-rise' vs. ,fall) haben Relevanz. Was die Syntax anbelangt, ist über den bereits erwähnten V2-Parameter hinaus der Direktionalitätsparameter aufgrund der Platzierung von Kopf und Komplement, d.h. der Verb-Objekt-Beziehung (d.h. die Unterscheidung von Sprachen mit VO-Basisgliedfolge und OV-Abfolge), aber auch die Flexibilität der Wortstellung und die Existenz von verschiedenen Konstruktionen wie „presentational“ und „existential constructions“, Satz-

Für die Markierung des hierarchischen Zusammenhanges wurde das Zeichen „, “ verwendet. Dieses Zeichen signalisiert, dass die einzelnen Kategorien der Hierarchie in einem implikativen Zusammenhang stehend aufzufassen sind. Die Möglichkeit bzw. Relevanz von $[+\mathrm{C}]-$ Kontrast impliziert die Relevanz von [+C]-Kontinuität, $[-\mathrm{C}]$ impliziert die Möglichkeit bzw. Relevanz von $[+\mathrm{C}]$-Kontrast und $[+\mathrm{C}]$-Kontinuität. 
spaltung usw. von Belang (siehe unten). Zuletzt ist auch der Einfluss verschiedener Parameter in der Pragmatik-Syntax-Schnittstelle wie der Diskurskonfigurationalitätsparameter mit Topik-Prominenz und Fokus-Prominenz (bzw. Topik- und Fokus-Konfigurationalität) zu nennen.

Die relevanten Konstruktionen, deren Präsenz und Häufigkeit bzw. Abwesenheit die Trennung der drei hierarchisch geordneten Kategorien von Sprachen motivieren, sind einerseits diejenigen Konstruktionen, die $[+\mathrm{C}]-$ Kontinuität am Satzanfang sichern, andererseits diejenigen, die die initiale Platzierung von $[+\mathrm{C}]-$ Kontrast-markierten Elementen fördern. Schließlich ist auch die Präsenz und Unmarkiertheit von Strukturen zu beachten, die die Anfangsstellung von nicht-C-bezogenen, so genannten [-C]-markierten Konstituenten ermöglichen.

Bei der erstgenannten Kategorie der Sprachen, für die die [+C]-Kontinuitätsmarkierung entscheidend ist, geht es darum, dass [-C]-markierte Elemente am Satzanfang vermieden werden. Dies ist vor allem durch die Anwendung von „presentational“ und „existential constructions“ und Satzspaltung (,"clefts") möglich, da in diesen Konstruktionen das [-C]-markierte Element nach rechts verschoben wird. Für die Sicherung der [+C]-Markiertheit und Vermeidung des negativen Wertes des C-Merkmals am Satzanfang sind aber nicht nur rechtsorientierte, sondern auch linksorientierte Operationen - wie Topikalisierung und Inversion - zuständig.

Zu den [+C]-Kontrast-sichernden Konstruktionen gehört vor allem die Satzspaltung. Wie oben schon erwähnt, wird das kontrastierte Element durch diese Konstruktion im Satz nicht nur nach vorne gerückt, sondern gleichzeitig auch nach rechts (siehe oben Abschnitt 2). Aber auch bestimmte Typen von linksorientierten Bewegungen können an die Kontrastmarkierung gebunden sein - wie z.B. Topikalisierung im Englischen (vgl. (51)). Auch derjenige Typ der Linksbewegung, der das Element über die initiale Satzgrenze hinaus bewegt und die Platzierung des akzentuierten Elements satzextern ermöglicht, ist in diesem Zusammenhang relevant. Dies ist bei der so genannten „Left dislocation“ (Linksherausstellung) der Fall.

Schließlich gehören zu den initialen $[-\mathrm{C}]$-realisierenden Konstruktionen thetische Sätze mit dem Nuklearakzent am Satzanfang, der dem Fokusexponenten zugewiesen wird, sowie Sätze mit einem satzinitialen „,presentational focus" (neuem Fokus) mit oder ohne Akzent.

\subsection{Zur „C-Hierarchie“ - Kategorie 1}

Für die beiden Sprachen der ersten Kategorie - für das Französische und Schwedische - ist die Dominanz der [+C]-Kontinuität bezeichnend. Hier stellt sich allerdings die Frage, aufgrund welcher Eigenschaft sich diese Sprachen zu der gleichen Kategorie zuordnen lassen. Meines Erachtens lässt sich die Verwandtschaft des Französischen und Schwedischen in die- 
sem Zusammenhang dadurch begründen, dass in diesen Sprachen die satzinitiale Konstituente nicht - oder nur selten - den Hauptakzent trägt und dass auch der sekundäre Akzent nur selten in Frage kommt. Das gemeinsame Merkmal kann demnach durch Rückgriff auf die Phonologie mit folgender Beschränkung angegeben werden: „Avoid accent on the left periphery of the sentence. "Trotz der gemeinsamen akzentvermeidenden Strategie am Satzanfang weisen jedoch das Französische und das Schwedische relevante Unterschiede auf - sowohl im Bereich der Phonologie als auch der Syntax: Während im Schwedischen die Akzentuierung im Satz nicht an bestimmte Positionen gebunden ist - in Vallduvís (1992) Terminologie „plastisch“ ist -, hat das Französische eine so genannte „,non-plastic intonation“. Was die Syntax anbelangt, ist im Französischen die Abfolge von Subjekt und Prädikat festgelegt, im Schwedischen dagegen können - dank der V2Eigenschaft dieser Sprache - auch andere Satzglieder als das Subjekt vor dem Prädikat am Satzanfang auftreten. Das Schwedische nutzt allerdings diese Möglichkeit in einem viel geringeren Maße aus als das Deutsche, was - wie unten näher diskutiert wird - auf den Einfluss anderer Parameter zurückzuführen ist.

Die Dominanz der [+C]-Kontinuität für die beiden Sprachen lässt sich vor allem unter Rekurs auf Konstruktionen nachweisen, die den positiven Wert der C-Kontinuität dadurch sichern, dass sie es ermöglichen, die mit $[-\mathrm{C}]$ markierten Elemente oder die Elemente mit dem [+C]-Kontrast-Merkmal am Satzanfang zu vermeiden. Verschiedene Fokustypen - der nichtkontrastive Informationsfokus in voll-fokussierten Sätzen (die eine allgemeine Informationsfrage „Was ist passiert?" beantwortet) oder der auf eine Konstituente beschränkte (kontrastive oder nicht-kontrastive) „,narrow focus" - stehen demnach in diesen Sprachen nicht oder nur relativ selten am Satzanfang. ${ }^{7}$ Wie Larsson Ringqvist (2003) in ihren kontrastiven Untersuchungen zum Französischen und Schwedischen gezeigt hat, ist besonders die hohe Frequenz der Satzspaltung in beiden Sprachen auffallend.

Die Satzspaltung kommt im gesprochenen Französisch in zwei Varianten vor - als c'est-cleft (mit der „exhaustiven“ Lesart) in (18) und als avoir-cleft (mit der „listing“ Lesart) in (19). Im gesprochenen Französisch ermöglicht diese Konstruktion auf eine optimale Weise die Nachstellung von initialem „narrow focus“, 8 der mit der „exhaustiven“ Lesart (18) auch kontrastiven Charakter hat (vgl. Lambrecht 1994):

$$
\begin{array}{ll}
\text { C'est les ENFANTS qui sont allés à l'ecole. } & \text { (exhaustive reading) } \\
\mathrm{Y} \text { a les ENFANTS qui sont allés à l'ecole. } & \text { (listing reading) }
\end{array}
$$

Diesen Fokustypen ist nämlich gemeinsam, dass sie auch den primären (obligatorischen) Akzent tragen oder ihnen für die Markierung der Topik-Kommentar-Gliederung ein sekundärer Akzent zugewiesen wird.

8 Die Akzente werden in diesen Beispielen ((18) und (19)) durch Majuskeln markiert. 
Beim kontrastiven Fokus ist auch im Schwedischen die Satzspaltung die präferierte Alternative. Dies zeigt das schwedische Beispiel (20), in dem Peter der im Kontext figurierenden Alternative Paul gegenübergestellt wird:

[Kontext: A: Jag tror att Paul kommer.] ,Ich glaube, dass Paul kommt.'

B: Nej. Det är PEter som kommer.'

,Nein. Es ist Peter, der kommt.'

(Huber 2002)

Die Satzspaltung wird aber sowohl im Französischen als auch im Schwedischen auch zur Nachstellung von, initialen weiten Foki“" (d.h. [-C]-markierter Elemente) verwendet. In diesen Fällen erstreckt sich die Fokusdomäne über den ganzen Satz und das satzinitiale Glied ist somit - wie in dem französischen Beispiel (21) und dem schwedischen Beispiel (22) - ein [-C]-markierter Informationsfokus. Durch die Satzspaltung wird in diesen maximal fokussierten ,kategorischen“ Sätzen die Topik-Kommentar-Gliederung verdeutlicht (vgl. Huber 2002):

(21) C'est avec plaisir que je vous invite à participier à ce séminaire. (Declerck 1984)

(22) Det var för tre år sedan (som) Sverige fick en ny statsminister. es war vor drei Jahren (dass) Schweden bekam einen neuen „Staatsminister“ (Huber 2002)

Die Satzspaltung ist in beiden Sprachen auch eine optimale Lösung für die Nachstellung des fokussierten Gliedes in thetischen Sätzen. Thetische Sätze enthalten - im Gegensatz zu den so genannten kategorischen Sätzen - nur ein Urteil, d.h. die Information ist nicht in Topik und einen sich darauf beziehenden Kommentar gegliedert. In solchen Sätzen wäre das Subjekt mit dem Nuklearakzent am Satzanfang problematisch - vor allem im Französischen. Durch die Satzspaltung wird aber das Problem in den französischen Beispielen (23), (24) und dem schwedischen Beispiel (25) ${ }^{10}$ eliminiert:

(23) C'est MAMAN qui me bat. (Sasse 1987)

(24) Y'a le TELEPHONE qui SONNE! (Lambrecht 1994)

(25) [Kontext: Peter ringde till Anna] ,Peter hat Anna angerufen.

Det var hennes MAN som svarade.

es war ihr Mann der antwortete (Huber 2002)

Für die Nachstellung vom [-C]-markierten Informationsfokus stehen aber auch andere Typen von Konstruktionen zur Verfügung. Für die Vermeidung

$9 \quad$ Der kontrastive Akzent wird in Beispiel (20-B) durch die Verwendung von Majuskeln in der akzentuierten Silbe markiert.

10 Die Akzente werden in diesen Beispielen ((23), (24) und (25)) durch Majuskeln markiert. 
von indefiniten Nominalphrasen werden die so genannten ,presentational“ und ,existential constructions“ sehr häufig verwendet, wie mit den französischen Beispielen (26), (27) und den schwedischen Beispielen (28), (29) illustriert wird:

Il y a un livre sur la table

(27) Il est entré un roi. (Lambrecht 1994)

(28) Det finns kräm i kylen.

„Es gibt Creme im Kühlschrank.' (SAG 1999/1)

(29) Det kommer en man.

,Es kommt ein Mann.' (SAG 1999/1)

Es ist allerdings wichtig, hervorzuheben, dass trotz der Dominanz der $[+\mathrm{C}]-$ Kontinuität am Satzanfang im Französischen und Schwedischen der [+]-CKontrast satzinitial nicht ausgeschlossen ist. Im Französischen kommt aber für den Ausdruck des [+]-C-Kontrasts nur eine satzexterne Position in Frage. Akzentzuweisung zu einer Konstituente in einer satzexternen Position - z.B. bei Linksherausstellung eines Topiks („Left Dislocation“) - wie in den Beispielen (30) oder (31) - ist demnach auch im Französischen völlig unproblematisch. Lambrecht (1994, S. 292) weist darauf hin, dass in kontrastiven Fällen im Französischen (in der gesprochenen Sprache) neben der „Left Dislocation“ auch die Satzspaltung häufig benutzt wird (vgl. (32)): ${ }^{11}$

(30) MOI je PAYE. (Lambrecht 1994)

(31) Maria prend l'avion pour Berlin (tandis que)

(,) PIERRE lui va à Stockholm en TRAIN.

(32) H: Mary, TOI tu fais les NETTOYAGES,

MOI je fais la CUISINE.

M: Non, c'est MOI qui fais la CUISINE,

TOI tu peux faire autre CHOSE.

(Lambrecht 1994)

Im Gegensatz zum Französischen ist im Schwedischen für die intonatorische Markierung des Kontrasts am Satzanfang die Linksherausstellung nicht zwingend: Hier kann das satzinterne Element am Satzanfang einen Akzent mit steigender Kontur (in Beispiel (33) durch " markiert) erhalten, was vor allem in denjenigen Fällen ausgenutzt wird, in denen das initiale Element Topik ist. ${ }^{12}$ Dies ist z.B. in Beispiel (33) der Fall:

$11 \quad$ Die Akzente werden in diesen Beispielen ((30), (31) und (32)) durch Majuskeln markiert.

12 Diese Fälle zeigen auch deutlich, dass Akzentzuweisung im Schwedischen am Satzanfang möglich ist und die Beachtung der Beschränkung „Avoid accent on the left periphery of the sentence" im Schwedischen nicht zwingend ist. Dies motiviert jedoch nicht die Zuordnung des Schwedischen zur Kategorie 2, da im Schwedischen der initiale Kontrastakzent (im 
(33) Jonas känner jag inte, men "Henrik har jag ofta pratat med. Jonas kenne ich nicht aber Henrik habe ich oft gesprochen mit (SAG 1999/4)

Die Satzspaltung ist aber auch im Schwedischen eine denkbare Alternative bei den kontrastiven Topiks, vgl. (34): ${ }^{13}$

[Kontext: A:

$$
\text { B: }
$$

Anna spelar piano och Max fiol. ,Anna spielt Klavier und Max Geige.

Nej. Det är MAX som spelar PiAno nein es ist Max der spielt Klavier (, och ANna som spelar fiOL). und Anna der spielt Geige (Huber 2002)

\subsection{Zur „C-Hierarchie“ - Kategorie 2}

Zu der zweiten Kategorie der C-Hierarchie gehören genetisch völlig unterschiedliche Sprachen, wie das Finnische und das Russische. Alles deutet darauf hin, dass in diesen Sprachen über die positiv markierte C-Kontinuität hinaus auch der positiv markierte C-Kontrast am Satzanfang wichtig bzw. grammatikalisiert ist, während [-C]-markierte Elemente entweder nicht zulässig sind oder nur selten in Frage kommen. Die Grundlage der Zugehörigkeit zu einer gemeinsamen Kategorie bildet vor allem die Morphologie, auch wenn hier syntaktische Eigenschaften eine gewisse Rolle spielen. Im Hinblick auf die Morphologie ist für beide Sprachen kennzeichnend, dass sie keine Artikel haben und somit bei der Kennzeichnung des informationsstrukturellen Status der Elemente nicht - oder kaum - auf morphologische Mittel rekurrieren können. Die Markierung des Topik- bzw. Fokuscharakters der Konstituenten wird allerdings durch die flexible Wortstellung ermöglicht.

Vielleicht ist es aber nicht völlig überraschend, dass das Finnische sich jedoch in mancher Hinsicht vom Russischen unterscheidet. Zum einen ist ein phonologischer Unterschied - das Fehlen verschiedener Akzenttypen (z.B. des steigenden Tonmusters) im Finnischen und das Vorliegen von „fall-rise“ im Russischen (vgl. Mehlhorn 2002) - hervorzuheben. Zum anderen ist bezüglich des syntaktischen Vergleichs relevant, dass im Finnischen der Kontrast am Satzanfang sowohl mit Topik- als auch mit Fokusfunktion (d.h. sowohl mit dem pränuklearen Akzent als auch mit dem Nuklearakzent) eine eigene Position beanspruchen kann (siehe unten die finnischen Beispiele (39) und (42), in denen das akzentuierte Element in der Kontrastposition Fokus (39) und Topik (42) ist).

Gegensatz zum Finnischen und Russischen) nicht nur markiert, sondern in der Regel vermieden wird.

13 Der kontrastive Akzent wird in Beispiel (34) durch die Verwendung von Majuskeln in der akzentuierten Silbe markiert. 
Gemeinsam ist also beiden Sprachen, dass die Markierung von [+C]Kontinuität durch syntaktische Mittel - durch die satzinitiale Platzierung des C-markierten Elements - relevant ist, da dies in keiner der Sprachen durch die Verwendung von Artikeln möglich ist. ${ }^{14}$ Im Finnischen können somit sowohl subjektinitiale und objektinitiale Sätze - Sätze mit SVO wie in Beispiel (35) und OVS wie in Beispiel (36) - als neutral betrachtet werden:

Esa luki kirjan. ,Esa las ein Buch.

(36) Kirjan luki Esa.

,Das Buch wurde von Esa gelesen.

(Järventausta 2003)

Auch im Russischen werden subjektinitiale und objektinitiale Sätze - wie (37) und (38) - als „,neutral basic variants“ bezeichnet (vgl. Zybatow/Junghanns 1998 und Krylova/Chavronina 1976) - vorausgesetzt, dass sich der Nuklearakzent ${ }^{15}$ weiter hinten im Satz befindet:

$$
\begin{aligned}
& \text { Predsedatel' otkryl sobranie. } \\
& \text { Vorsitzender-nom eröffn-Prät/sg/mask Versammlung-akk } \\
& \text {,Der Vorsitzende eröffnete die Versammlung.' }
\end{aligned}
$$

Kartu prineset laborant.

Karte-akk bring-Präs/3P/Sg Laborant-nom

,Die Karte bringt der Laborant.'

Die Realisierung des Kontrasts am Satzanfang ist aber in beiden Sprachen durchaus möglich und im Gegensatz zum Französischen (und Schwedischen) wird sie auch nicht durch unterschiedliche Strategien blockiert. Ganz wesentlich ist aber zu betonen, dass im Finnischen die Platzierung eines Elements in der satzinitialen KONTRAST-Position die prototypische Variante der Kontrastivität - d.h. das Vorliegen von kontextuell gegebenen Varianten - verlangt. Diese Bedingung wird in Beispiel (39) durch die Gegenüberstellung von Stockholm und Reykjavik erfüllt. Wie Beispiel (40) zeigt, kann aber ein finnischer FOCTOP-Satz, ${ }^{16}$ in dem die KONTRAST-Position vom Fokus besetzt ist, nicht als eine adäquate Antwort auf eine Ergänzungsfrage betrachtet werden, wenn die $W$-Frage nur die Menge angibt, die relevanten Alternativen aber nicht spezifiziert werden:

14 Die Markierung von $[+\mathrm{C}]$-Kontinuität wird aber durch adnominale Demonstrativa ermöglicht.

15 Der Nuklearakzent wird in den russischen Beispielen ((37) und (38)) durch Fettdruck markiert.

16 Vgl. Vilkuna (1995, S. 249), die im Finnischen zwei Satzmuster unterscheidet: FOCTOP-Sätze und TOP-Sätze. Während in den FOCTOP-Sätzen die satzinitiale Kontrast-Position vom Fokus besetzt ist, enthält diese Position in den TOP-Sätzen das Topik. 
(39) A: Pekka lensi Tukholmaan.

Pekka flog nach Stockholm

B: [kontrast Reykjavikiin] Pekka lensi. ${ }^{17}$

nach Reykjavik Pekka flog

(40) F: Where did Pekka fly to?

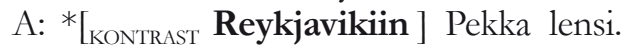

nach Reykjavik Pekka flog (Molnár/Järventausta 2003)

Auch bezüglich des Russischen wird behauptet, unter anderem in einem kürzlich erschienenen Artikel von Neeleman/Titov (2009), dass die Basisposition eines fokussierten Elements die Letztposition ist, dass aber das Kontrastmerkmal - und nur das Kontrastmerkmal - die Bewegung an den linken Satzrand lizenziert.

Varianten mit dem fokussierten Glied am Satzanfang werden im Russischen aber nur als „expressive Varianten“ bezeichnet - der prototypische Kontrast scheint jedoch den Angaben in der Literatur zufolge keine notwendige Bedingung für die Spitzenstellung des Fokus zu sein. Laut einer Informantenbefragung haben russische Muttersprachler die Antwort auf eine $W$-Frage (41-A), in der das Ziel der Reise nachgefragt wurde, mit der Anfangsstellung des fokussierten Elements in (41-B-b) adäquat gefunden. Eine solche Alternative wurde - wie ich oben gezeigt habe - im Finnischen als ungrammatisch beurteilt. Die Wahl der satzinitialen Konstituente Jalta, eines bekannten Elements, kann allerdings aufgrund der [+C]-Kontinuitätsmarkiertheit auch zur Legitimierung der Antwort in (41B-b) beigetragen haben: ${ }^{18}$

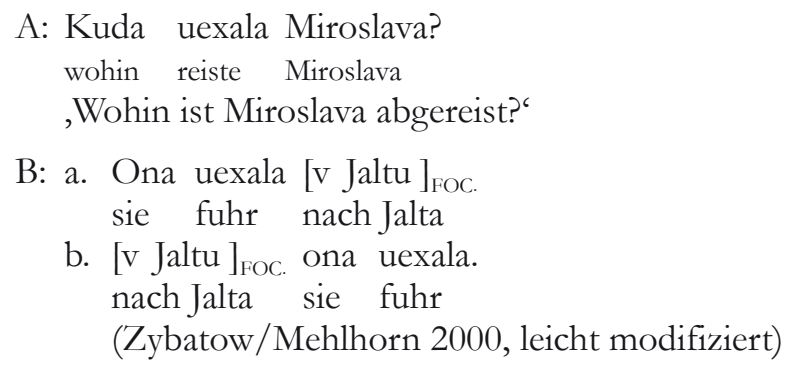

Auch Topiks sind mit dem [+C]-Kontrast-Merkmal verträglich. Im Finnischen können aber in der KONTRAST-Position nur prototypische Kontrasttopiks - mit kontextuell gegebenen Gegenalternativen - stehen. Dies

\footnotetext{
17 Der kontrastive Akzent wird in den finnischen Beispielen (39) und (40) durch Fettdruck markiert.

18 Ich möchte dem unbekannten Reviewer für die Kommentierung dieser Beispiele danken. $\mathrm{Er} / \mathrm{sie}$ weist unter Rekurs auf Informanten darauf hin, „dass b. eine spezifischere Bedeutung als a. hat. B ist im Sinne von ,Jalta wie du weißt' zu verstehen“". Dies scheint auch die Annahme zu bestätigen, dass in diesem Falle die Bekanntheit des erstgenannten Gliedes von Belang ist.
} 
wird mit den so genannten TOP-Sätzen $(42)^{19}$ illustriert (in diesen Sätzen steht das Topik mit dem pränuklearen Akzent am Satzanfang, siehe oben Fußnote 16):
a. [KOntrast
Tukholmaan] Pekka lensi nach Stockholm Pekka flog
Finnairilla]. ,Nach Stockholm, Pekka flog mit Finnair.'
b. [Kontrast Reykjavikiin] (Pekka lensi) [focus Icelandairilla]. nach Reykjavik (Pekka flog) mit Icelandair ,Nach Reykjavik (Pekka flog) mit Icelandair. (Molnár/Järventausta 2003)

Wie die experimentell getesteten Daten von Mehlhorn (2002, S. 63) zeigen, ermöglicht im Russischen der (fallend-)steigende „(fall-)rise“-Akzent die Signalisierung des kontrastiven Topiks am Satzanfang, vgl. Beispiel (43b): ${ }^{20}$
a. Ja slysal, chudožnik zakončil rabotu ich hörte der Künstler beendete die Arbeit ,Ich habe gehört, dass der Künstler die Arbeit beendete.
b. Por/TRET o KONčen, nat'ur/MORT jesče n'e goTOV. das Portrait abgeschlossen das Landschaftsbild noch nicht fertig ,Das Portrait ist abgeschlossen, das Landschaftsbild ist noch nicht fertig."

\subsection{Zur „C-Hierarchie“ - Kategorie 3}

In der dritten Kategorie der C-Hierarchie scheint die Realisierung von Elementen mit dem [-]-C-Merkmal am Satzanfang bedeutend leichter zu sein als in den bisher diskutieren Sprachgruppen. Selbstverständlich sind auch in diesen nicht-verbinitialen Sprachen die Alternativen [+C]-Kontinuität und [+]-C-Kontrast relevant. Unter diese dritte Kategorie wurden das Englische, Deutsche und das Ungarische subsumiert - drei Sprachen, die sich in vielerlei Hinsicht im Hinblick auf eine Reihe von Parametern unterscheiden:

(i) Das Deutsche ist im Gegensatz zu den beiden anderen Sprachen eine Verb-zweit-Sprache (V2);

(ii) Für das Englische ist Subjektprominenz und „rigid word order“ charakteristisch, während die Wortstellung in den beiden anderen Sprachen (vor allem die Stellung der Argumente und freien adverbialen Angaben) flexibel ist;

\footnotetext{
19 Der kontrastive Akzent wird in den finnischen Beispielen (42a) und (42b) durch Fettdruck markiert.

20 Der kontrastive Akzent wird in Beispiel (43) durch die Verwendung von Majuskeln in der akzentuierten Silbe markiert.
} 
(iii) Im Ungarischen ist die Position des Fokus fest geregelt - Ungarisch wird als eine fokuskonfigurationale Sprache beschrieben;

(iv) Expletive Pronomen - und damit zusammenhängend „clefts“, „presentational“ und „existential constructions" - sind im Englischen und Deutschen möglich, im Ungarischen kommen dagegen diese Konstruktionen nicht vor;

(v) Auch die phonologischen Unterschiede bezüglich der Direktionalität der NSR - „righthand-NSR“ vs. „lefthand NSR“ - sind auffallend, im Ungarischen ist die NSR im Gegensatz zum Deutschen und Englischen linksorientiert.

Da eine Analyse der oben erwähnten drei Sprachen im Hinblick auf sämtliche relevante Parameter den Rahmen dieser Arbeit sprengen würde, sollen an dieser Stelle nur ein paar ausgewählte Aspekte des Sprachvergleichs angesprochen werden. Dabei möchte ich die Trennung dieser Gruppe von den anderen Kategorien der C-Hierarchie motivieren und auch die interne Rangordnung innerhalb der Gruppe kurz andeuten.

Für die Trennung der dritten Kategorie von den ersten beiden Kategorien der Hierarchie ist vor allem die Möglichkeit der Platzierung von [-C]markierten Elementen in Betracht zu ziehen. Ein [-C]-markiertes Element ist in allen drei Sprachen möglich - und sogar unmarkiert - am Satzanfang von thetischen Sätzen, in denen das satzinitiale Element auch den nuklearen Akzent trägt und somit als so genannter Fokusexponent im Satz fungiert. Dies wird mit dem englischen Satz (44b), dem deutschen Satz (45) und den ungarischen Beispielen (46) und (47) illustriert:

(44) a. What's this noise?

b. The CAT is miaowing. (Sasse 1987)

$$
\text { Die SONne scheint. }{ }^{21}
$$

$$
\begin{aligned}
& \text { 'Ugat a 'kutya. }{ }^{22} \\
& \text { bellt der Hund } \\
& \text { 'Iskolába 'mentek a 'gyerekek } \\
& \text { zur Schule gingen die Kinder }
\end{aligned}
$$

Möglich ist auch die initiale Platzierung eines [-C]-markierten Elements in vollfokussierten - in der Regel diskursinitial auftretenden - Sätzen. Vollfokussierte Sätze verlangen allerdings in diesen Fällen in allen drei Sprachen

${ }^{21}$ Der Nuklearakzent wird in den russischen Beispielen (44) und (45) durch Majuskeln markiert.

22 In den ungarischen Beispielen (46) und (47) wird die so genannte „level prosody“ durch die Verwendung des Zeichens ,"“vor der akzentuierten Silbe markiert. 
das Subjekt am Satzanfang. Der Informationsfokus kann in diesen drei Sprachen durch den indefiniten Artikel markiert werden (vgl. die Beispiele $(48)-(50)):^{23}$

(48) A wizard once was very wise, rich, and married to a beautiful witch. (Lambrecht 1994)

(49) Ein Kind hat einen HAsen gefangen. ${ }^{24}$ (Fanselow/Lenertová 2011)

(50) Egy egyetemista kereste az igazgatót telefonon. ein Student suchte den Direktor am Telephon

Die Unterschiede innerhalb der dritten Kategorie sind aber nicht unwesentlich und verlangen dadurch eine weitere Hierarchisierung der Sprachen. Besonders im Englischen ist die Voranstellung von anderen Satzgliedern außer Subjekten abhängig von der C-Markiertheit des jeweiligen Gliedes, wobei sich der Einfluss der Kontrastmarkierung besonders deutlich zeigt. Bei der Topikalisierung eines fokussierten Elements ${ }^{25}$ ist nämlich im Englischen das Vorliegen eines kontextuell verankerten Kontrasts eine Voraussetzung (51):

a. They named their dog [F FIDO]

b. [F FIDO ] they named their dog.

Beispiele (51a) und (51b) kommentierend, behaupten Vallduví und Engdahl (1996, S. 475):

In these example the specification of the relevant value is indeed focal and triggers the appropriate structural realization by means of prosody, but the scale this value belongs to is part of the background. In fact, it is a link, and this is what triggers its realization as a fronted element. Crucially, Fido in ([51]-a) does not give rise to the same interpretation.

Im Englischen liegen auch [+]-C-Kontrast-bezogene Restriktionen beim Topik vor: Linksherausgestellte „Left-dislocated“ (LD)-Topiks und Topiks, die durch (satzintern durchgeführte) Topikalisierung (TOP) ${ }^{26}$ an die Satz-

23 Trotz des engen Zusammenhanges zwischen dem indefiniten Artikel und dem Fokus ist der indefinite Artikel nicht an die Fokuslesart gebunden bzw. die Fokuslesart verlangt nicht die Verwendung des indefiniten Artikels.

24 Der Nuklearakzent wird in Beispiel (49) durch die Verwendung von Majuskeln in der akzentuierten Silbe markiert.

25 Der Fokusakzent wird in den englischen Beispielen (51a) und (51b) durch Fettdruck markiert.

26 Der Terminus „Topikalisierung“ (TOP) bezieht sich auf eine strukturelle Operation im Englischen, die nicht an die Topik-Funktion gebunden ist. Reinhart (1982) zufolge ermöglicht die Topikalisierung der Nominalphrase your second proposal in (V-a) und der Präpositionalphrase with Rosa in (V-b) sowohl die Topik-Lesart als auch die Fokus-Interpretation. 
spitze geraten, sind in Antworten auf allgemeine Informationsfragen nicht adäquat. Ihre Unangemessenheit wird von Prince (1984) in den Antworten (52b) und (52c) entsprechend markiert:

$$
\text { Q: What did you do over vacation? }
$$

a. A: I read two books.

b. A: \# Two books I read.

c. A: \# Two books, I read them. [LD] (Prince 1984)

Dieses Problem ist allerdings beim Vorliegen des Kontrasts in den unten stehenden Beispielen für Topikalisierung - entweder bei der Implikation eines Gegensatzes wie in Beispiel (53a/B) oder bei der Realisierung eines Kontrasts wie in Beispiel (53b/B) - nicht vorhanden:

a. A: You want to see Annie Hall?

B: Annie Hall I saw yesterday.

b. A: You see every Woody Allen movie as soon as it comes out.

B: No - Annie Hall I saw (only) yesterday. (Prince 1984)

Die Angemessenheit von „Left Dislocation“ mit kontrastierten Elementen wird von Prince (1984) mit Beispiel (54) illustriert, in dem verschiedene Gesundheitsprobleme wie gallstones und nervous breakdown einander gegenübergestellt werden:

(54) „Everybody has their little bundle, believe me. I'll bet she had a nervous breakdown. That's not a good thing. Gallstones, you have them out and they're out. But a nervous breakdown, it's very bad ..." (Ref. 21, p. 162)“ (Prince 1984)

Die Sicherung der [+]-C-Kontinuität am Satzanfang ist im Englischen ebenfalls relevant - dazu werden aber aufgrund der Rigidität der Wortstellung besondere Strategien verwendet, wie z.B. die Inversion. Die Inversion ist in Birners Formulierung (1994, S. 234, 236, 245) ,an information-packaging mechanism, allowing the presentation of relatively familiar information before a comparatively unfamiliar logical subject" ". Der Referent der kursivierten Phrase them in Beispiel (55) ist im vorangehenden Kontext schon erwähnt (loud-speakers), der postverbale Teil enthält aber neue Information:

Loud-speakers had been erected along the garden: < through them $>$ emerged $<$ the bland informal voice of Commander Stephen KingHall describing the scene as he saw it from the St. Paul's Cathedral. >

Vgl. Reinhart (1982, S. 10): „NP’s fronted by topicalization, as in [V-a] or by PP preposing, as in [V-b] can receive focus (i.e. non-topic or ,new information') intonation, in which case they will not serve as topics, but without this intonational mark they must be interpreted as topics.

(v) a. Your second proposal, the board found unfeasible.

b. With Rosa, Felix went to the beach." 
Birner (1994, S. 245) weist auch darauf hin, dass „the opposite ordering - a preposed discourse-new element with a postposed discourse-old element - results in infelicity". Dies wird in Beispiel (56-B) gezeigt:

(56) A: Hey, mom, have you seen my gym shirt? I'm in a big hurry to get to the bus stop.

B: \#In the hall closet is your gym shirt. (cf. Your gym shirt is in the hall closet.) (Birner 1994, S. 245)

Im Gegensatz zum Englischen gibt es im Ungarischen bei der Realisierung des Fokus und des Topiks keine bzw. nur schwache C-Merkmal-bezogenen Restriktionen am Satzanfang. Dies lässt sich auf folgende strukturelle Besonderheiten des Ungarischen zurückführen: (i) auf die Fokuskonfigurationalität des ungarischen Satzes (mit Realisierung des Fokus in einer designierten Position) in Kombination mit der „pro-Drop“-Eigenschaft, die häufig zum so genannten „Topik-Drop“ führt und die satzinitiale Realisierung des Fokus ermöglicht; (ii) auf die bereits angesprochene satzinitiale Platzierung des Fokus in thetischen Sätzen, in denen die nichtverbalen Konstituenten in situ (postverbal) stehen können; sowie (iii) auf die Linksorientiertheit der Nuclear Stress Rule (NSR), die die Unmarkiertheit des satzinitialen Akzents erklärt. Besonderes Interesse verdient in diesem $\mathrm{Zu}$ sammenhang auch die Tatsache, dass im Ungarischen der Fokus nicht notwendigerweise kontrastiv - d.h. C-bezogen - ist: In mehreren Arbeiten wird dafür argumentiert (vgl. Molnár 1998; Horvath 2007), dass bei der ungarischen Fokusbewegung nicht die pragmatische Dimension der Fokussierung (als ,neue Information“) relevant ist, sondern die semantische Verankerung des fokussierten Gliedes in einer Alternativenmenge.

Wie Gécseg/Kiefer (2009) in ihrem Artikel zeigen, sind im Ungarischen die C-bezogenen Restriktionen auch beim Topik nicht entscheidend. In den unten zitierten Beispielsätzen (57) und (58) kann sowohl das indefinite Subjekt als auch das indefinite Objekt satzeinleitend stehen. Ganz wesentlich ist dabei, dass beide Sätze (und nicht nur der Satz mit dem Subjekt am Satzanfang) eine allgemeine Informationsfrage wie Was ist passiert? beantworten können, was darauf hindeutet, dass sich in diesen Beispielsätzen die Fokusdomäne über den ganzen Satz - somit auch über die satzinitiale Position - erstreckt: ${ }^{27}$

(57) Egy 'kutya 'megharapott egy 'járókelót. ein Hund perf-biss einen Fussgänger ,Ein Hund biss einen Fussgänger.

(58) Egy'járókelôt 'megharapott egy 'kutya. einen Fußgänger perf-biss ein Hund ,Einen Fußgänger biss ein Hund.' (Gécseg/Kiefer 2009)

27 Die so genannte „level prosody“ wird in den ungarischen Beispielen (57) und (58) durch die Verwendung des Zeichens ,"“ vor den akzentuierten Silben markiert. 


\section{Deutsch im Sprachvergleich}

Wie oben angenommen (vgl. hierzu die C-Hierarchie in (17)), nehmen in der dritten Kategorie der Hierarchie das Englische mit wesentlichen C-bezogenen Restriktionen und das Ungarische mit einer relativen Unabhängigkeit von der C-Markierung die beiden Extrempositionen ein. Dies impliziert gleichzeitig, dass sich das Deutsche zwischen diesen beiden Extrempolen befindet. Im abschließenden Teil des vorliegenden Aufsatzes wird die Aufmerksamkeit auf das Deutsche gerichtet, das zum einen den beiden anderen Sprachen der dritten Kategorie gegenübergestellt, und zum anderen mit einer eng verwandten Sprache, dem Schwedischen, verglichen wird.

Bei dem „kategorieinternen“" Vergleich des Deutschen mit dem Englischen und dem Ungarischen lässt sich festhalten, dass das Deutsche im Gegensatz zum Englischen - aufgrund seiner V2-Eigenschaft einen größeren Spielraum für die Spitzenstellung von unterschiedlichen Konstituenten eröffnet (siehe oben die Beispiele (1)-(6)), und dass die Topikalisierung von Objekten, Prädikativen und Adverbialen nicht notwendigerweise Kontrastivität voraussetzt bzw. zur Folge hat (vgl. hierzu auch Speyer 2004, 2008). Die kontextuelle Gegebenheit der Konstituenten (d.h. ihre [+C]-Markiertheit) scheint allerdings auch in diesen „nicht-kontrastrelatierten“ Fällen ein wichtiger Faktor für die Bewegung an den Satzanfang im Deutschen zu sein (siehe unten den Vergleich des Deutschen und des Schwedischen).

Es ist allerdings zu betonen, dass die Vorfeldbewegung im Deutschen nicht immer von diskursbezogenen Merkmalen motiviert zu sein braucht - auch Konstituenten ohne [+]-C-Markierung können an den Satzanfang geraten. Wie die nach Fanselow/Lenertová (2011) zitierten Beispiele (59) und (60) zeigen, sind nicht nur Sätze mit der satzinitialen Platzierung von indefiniten Subjekten wie in dem bereits oben zitierten Beispiel (49) angemessen als Antwort auf eine allgemeine Informationsfrage wie What's new?, sondern auch Sätze mit einem Satzadverbial (59) oder sogar mit einem indefiniten Objekt (60) am Satzanfang. In diesen Fällen bildet die satzinitiale Konstituente einen Teil der maximalen Fokusdomäne: ${ }^{28}$

Wahrscheinlich hat ein Kind einen HAsen gefangen.

(60) [Einen HAsen $]_{i}$ habe ich $\mathrm{t}_{i}$ gefangen.

Die satzinitial platzierte fokussierte Konstituente einen Hasen in (60) kann aber nicht nur in einem „vollfokussierten Satz“ vorkommen, sondern auch Teil einer auf die VP beschränkten Fokusdomäne sein - als Antwort auf die Frage What did you do? (Selbstverständlich ist auch die sich nur auf die NP beziehende enge Fokuslesart möglich.) In den erstgenannten Fällen geht es

28 Der Nuklearakzent wird in den deutschen Beispielen (59) und (60) durch die Verwendung von Majuskeln in der akzentuierten Silbe markiert. 
allerdings um eine Bewegung eines innerhalb einer größeren Fokusdomäne liegenden Elements, um einen so genannten SFF (,subpart of focus fronting“), d.h. nicht um den semantisch verankerten Fokustyp (,identificational focus", siehe oben) wie in fokuskonfigurationalen Sprachen (z.B. Ungarisch). Die Vorfeldbewegung wird somit nicht von diskurssemantischen Merkmalen wie F (Fokus) oder T (Topik) ausgelöst bzw. lizenziert. Fanselow/Lanertová (2011) behaupten, dass diese Daten dafür sprechen, dass der informationsstrukturelle Status des satzinitialen Elements für die Vorfeldbewegung irrelevant ist und dass sich diese Operation ausschließlich formal (von einem so genannten „edge feature ") motivieren lässt: „the left periphery is not confined to topics and foci in Czech and German, and [...] movement to SpecCP is not triggered or licensed by features related to information structure" (ebd., S. 174). Auch wenn dieser Grundannahme in der vorliegenden Arbeit nicht zugestimmt werden kann, ${ }^{29}$ ist in unserem Zusammenhang besonders die Beobachtung von Relevanz, dass die Topikalisierung von indefiniten Objekten mit einem gleichzeitigen Satzfokus oder VP-Fokus - d.h. ohne kontextuelle Verankerung, C-Markierung - wie in Beispiel (60) auch im Deutschen möglich ist. Dies zeigt, dass im Deutschen der positive Wert des C-Merkmals (der C-Kontinuität oder des C-Kontrasts) - ähnlich wie im Ungarischen - keine notwendige Bedingung für die Spitzenstellung ist.

Beim Vergleich des Deutschen mit dem Ungarischen wird jedoch deutlich, dass sich die C-bezogenen Restriktionen im Ungarischen in einem geringeren Maße als im Deutschen geltend machen. Der Unterschied lässt sich auf die unterschiedlichen Bedingungen von Pronomen-Dropping zurückführen. Im Ungarischen - einer „pro-drop-Sprache“ - gibt es keinen Zwang bezüglich der Realisierung einer pronominalen (präfokussalen) Konstituente: Das finite Verb am Satzanfang kommt nicht nur bei thetischen Sätzen vor (siehe oben die Beispielsätze (46) und (47)), sondern auch in vielen kategorischen, d.h. in Topik und Kommentar aufgeteilten Sätzen, wo aber das Topik (nicht nur das Subjekt, sondern auch das Objekt) ausgelassen werden kann. Sätze mit dem finiten Verb (wobei es als Fokus fungiert oder Teil des Fokusbereichs bildet) am Satzanfang sind somit im Ungarischen ziemlich frequent. Das Deutsche dagegen ist eine „Topik-drop-Sprache“ (vgl. Fries 1988) und die Weglassung von Pronomen (Subjekten, Objekten) ist stark restringiert: Im (gesprochenen) Deutschen können Subjekte und Objekte nur im Vorfeld gedroppt werden (61-B1), im Mittelfeld dagegen nicht. ${ }^{30}$

Siehe hierzu auch Frey (2010).

30 Vgl. Fries (1998, S. 24):

(vi) F. Hat Peter das Buch Fritz geschenkt?

$A_{1}$. Ja, hat er ihm geschenkt.

$A_{2}$. * Ja, er hat ihm geschenkt. 
Über den kategorieinternen Vergleich des Deutschen mit dem Englischen und Ungarischen hinaus möchte ich in den folgenden Überlegungen auf die einleitend gestellte Frage zurückkommen, nämlich auf die Frage, warum das Deutsche von einer eng verwandten Sprache - dem Schwedischen - bezüglich der Relevanz des Kohärenzprinzips abweicht. Beide Sprachen sind V2-Sprachen, wurden jedoch in der C-Hierarchie verschiedenen Kategorien zugeordnet. Aufgrund ihres V2-Charakters ist anzunehmen, dass die Topikalisierung in ihnen strukturell nicht restringiert ist. Es zeigt sich allerdings beim Vergleich dieser Sprachen, dass Topikalisierung im Schwedischen bedeutend stärker eingeschränkt ist als im Deutschen: Im schwedischen Fundament werden die Subjekte und die Pronomina stark bevorzugt. Weiterhin ist im Schwedischen die Topikalisierung anderer Satzglieder - z.B. der Objekte und bestimmter Adverbiale - mit einem kontrastiven Effekt verbunden. Im Deutschen dagegen ist die Frequenz der Objekte und Adverbiale im Vorfeld höher und die Topikalisierung dieser Elemente ist nicht unbedingt kontrastiv. Die Vorfeldbewegung kann entweder durch eine [+C]-Kontinuitätsmarkierung („Bekanntheit", „Erwähntheit") der Elemente motiviert sein; wie oben gezeigt ist aber auch die [-C]-Alternative denkbar.

Diese Unterschiede zwischen dem Deutschen und dem Schwedischen lassen sich auf den Einfluss weiterer struktureller Parameter zurückführen, vor allem auf den Einfluss des Direktionalitätsparameters bezüglich der VerbObjekt-Abfolge. Während das Deutsche eine OV-Sprache ist, ist die Basisgliedfolge im Schwedischen VO. Dieser Unterschied wirkt sich auf die Umstellungsmöglichkeiten im deutschen Mittelfeld bzw. im mittleren Bereich des schwedischen Satzes aus, und hat seinerseits auch Konsequenzen für die weiteren Bewegungsmöglichkeiten der Elemente ins schwedische Fundament und ins deutsche Vorfeld. Die Tatsache, dass im deutschen Mittelfeld die Linksbewegung von Objekten und bestimmten Adverbialen durch „Scrambling" - d.h. eine relativ freie Voranstellung kontextuell gegebener Elemente - möglich ist, und im Schwedischen Mittelfeld nur die Voranstellung von Pronomina - der so genannte „Object Shift“ - in Frage kommt, beeinflusst das informationsstrukturelle Potenzial der linken Peripherie.

Aufgrund der Scramblingmöglichkeit im Deutschen kann das Objekt oder ein Adverbial - wie in Beispiel (61) mit der Axt aus seiner Basisposition in (61a) an die Spitze des Mittelfelds geraten und dort vor dem Subjekt stehen (61b):

(61) a. (dass) Otto mit der Axt den Baum gefällt hat

b. (dass) mit der Axt Otto $_{1}$ den Baum gefällt hat SCRAMB

c. Mit der Axt $t_{1}$ hat ${ }_{1} t_{1}$ Otto $t_{1}$ den Baum gefällt FM (Frey 2006)

Die weitere Bewegung des Objekts oder des Adverbials ins Vorfeld (wie oben in (61c)) ist dann nur eine formale Angelegenheit - eine so genannte 
„formale Bewegung“" (vgl. Frey 2006). ${ }^{31}$ Wesentlich ist demnach in diesen Fällen, dass die Vorfeldfüllung den informationsstrukturellen Status der Konstituente nicht ändert und auch eine nicht-kontrastive, phonologisch unmarkierte $([+\mathrm{C}]-$ Kontinuität $]$-markierende) Interpretation des Adverbials mit der Axt am Satzanfang zulässt.

Frey zeigt auch, dass die „formale Bewegung“ im Deutschen nicht uneingeschränkt durchführbar ist. In einigen Fällen - bei bestimmten Adverbialen und Prädikativen wie in (62a) und (62b) - ist nämlich Scrambling nicht möglich. In diesen Fällen kann das satzinitiale Element nur als Resultat einer „genuinen“ A-bar-Bewegung die satzinitiale Position einnehmen, vgl. (63a) und (63b): ${ }^{32}$
a. *dass Maria grün die Tür $\mathrm{t}_{\iota}$ streichen wird
b. *dass Otto unfreundlich, sehr oft $\mathrm{t}_{\imath}$ gewirkt hat (Frey 2006)
a. GRÜN, wird Maria die Tür $t_{t}$ streichen
b. UNFREUNDLICH ${ }_{\imath}$ hat Otto sehr oft $\mathrm{t}_{\imath}$ gewirkt (Frey 2006)

Dieser Bewegungstyp hat aber laut Frey eine kontrastive Interpretation des satzinitialen Elements zur Folge: „In German, a long-moved topic receives a contrastive interpretation" (ebd., S. 245).

Im Gegensatz zum Deutschen ist im Schwedischen die formale Bewegung ins Fundament nur bei dem Subjekt und den Pronomina möglich, da die Umstellungsmöglichkeiten im mittleren Bereich des Satzes stark begrenzt sind. Nur die Pronomina können linksbewegt werden und der Negation vorausgehen, was in Beispiel (65) gezeigt wird: Hier ist nur die Voranstellung des Pronomens den grammatisch, im Gegensatz zur Voranstellung des Nomens bilen (den Wagen):

(64) Jag köpte inte bilen /den.

ich kaufte nicht den Wagen/den

,Ich habe den Wagen/den nicht gekauft.

(65) Jag köpte den /*bilen inte.

OBJECT SHIFT

ich kaufte den / den Wagen nicht

,Ich habe den/den Wagen nicht gekauft.

31 Die „formale Bewegung“ wird von Frey (2006, S. 241) folgendermaßen definiert: „[СP XP $\left[{ }_{C}, V_{\text {fin }}[\right.$ II $\left.\left.\ldots]\right]\right]$ can be derived from [ ${ }_{C}$ [ $\left.\left.{ }_{I P} \mathrm{XP} \ldots \mathrm{V}_{\text {fin }}\right]\right]$ (by moving the finite verb to $\mathrm{C}$ and) by moving XP as the highest element of IP to SpecCP (Minimal Link) just in order to fulfil the EPP requirement associated with $C$, i.e., without any additional intonational, semantic or pragmatic effects."

32 Der kontrastive Akzent wird in den deutschen Beispielen (63a) und (63b) durch Majuskeln markiert. 
Dies führt dazu, dass im Schwedischen im Gegensatz zu Subjekten (als DPs oder Pronomina) Nicht-Subjekte nur in pronominaler Form an den Satzanfang „formal bewegt" werden können (vgl. (66) und (67)). Alle anderen Nominalphrasen setzen - wie in Beispiel (68) eine genuine A-bar-Bewegung ins Fundament voraus, die immer kontrastiv ist: ${ }^{33}$

$\begin{array}{llll}\text { (66) } & \begin{array}{l}\text { Peter/PETER } \\ \text { Peter }\end{array} & \begin{array}{l}\text { köpte bilen } \\ \text { kaufte }\end{array} \text { den Wagen } & \begin{array}{l}\text { igår. } \\ \text { gestern }\end{array} \\ \text { (67) } & \begin{array}{l}\text { Den/DEN köpte Peter igår. } \\ \text { den }\end{array} \\ \text { kaufte } & \text { Peter gestern } \\ \text { (68) } & \text { *Bilen/BILEN } & \text { köpte Peter igår. } \\ \text { den Wagen } & \text { kaufte Peter gestern }\end{array}$

Die Bewegung ins schwedische Fundament ist somit in syntaktischer Hinsicht bedeutend aufwendiger als die Bewegung ins deutsche Vorfeld. Dies kann eine Erklärung dafür geben, warum im Schwedischen solche Bewegungen restringiert und nur unter bestimmten kontextuellen (kontrastbezogenen) Umständen lizenziert werden.

\section{Schlusswort}

Die Schwerpunkte dieses Beitrags lassen sich folgendermaßen zusammenfassen:

(i) Nicht der Topik- oder Fokusstatus der Elemente ist für ihre linksperiphere Platzierung relevant, sondern ihre „C-Markiertheit“.

(ii) Als universelle informationsstrukturelle Strategie wurde die Abfolge: „[+]-C-markiert]“ (Topik oder Fokus) vor „,[-C-markiert]“ (Topik oder Fokus) angegeben.

(iii) Die sprachspezifischen Unterschiede wurden auf die C-Hierarchie rekurrierend beachtet, und für die Erfassung der Variation in nicht-verbinitialen SV-Sprachen wurden drei Kategorien (mit weiterer interner Differenzierung) vorgeschlagen.

(iv) Das Deutsche wurde der dritten Kategorie zugeordnet, in der trotz der Relevanz des positiv markierten C-Merkmals auch [-C]-markierte Elemente am Satzanfang stehen können.

Es ist besonders hervorzuheben, dass bei der Aufstellung IS-relevanter Typen der Sprachen und dem Vergleich des Deutschen mit anderen Sprachen auf relevante strukturelle Besonderheiten und Parameter Bezug ge-

33 Der kontrastive Akzent wird in den schwedischen Beispielen (66), (67) und (68) durch Majuskeln markiert. 
nommen und somit die enge Beziehung zwischen informationsstrukturellen Strategien und strukturellen Regularitäten beachtet wurde. Die Analyse zeigte, dass sich die kognitiv und kommunikativ verankerten Prinzipien der Informationsstrukturierung nur von strukturellen Faktoren ermöglicht bzw. durch diese restringiert durchsetzen können. Die vorliegende Arbeit basiert demnach auf einem „Interface“-Ansatz, in dem - trotz der Anerkennung der Eigenständigkeit verschiedener sprachlich relevanter Module - das enge Verhältnis zwischen Grammatik und Pragmatik betont wird.

\section{Quelle}

Thomas Mann (1971): Die Bekenntnisse des Hochstaplers Felix Krull. Berlin/Weimar.

\section{Literatur}

Beauzée, Nicolas (1767): Grammaire générale, ou exposition raisonnée des éléments nécessaires du langage, pour servir de fondement à l'étude de toutes les langues. Paris.

Behaghel, Otto (1932): Deutsche Syntax. Eine geschichtliche Darstellung. Bd. 4: Wortstellung. Periodenbau. Heidelberg.

Birner, Betty J. (1994): Information status and word order: an analysis of English inversion. In: Language 70, 2, S. 233-259.

Chafe, Wallace L. (1974): Language and consciousness. In: Language 50, S. 111-133.

Chafe, Wallace L. (1976): Givenness, contrastiveness, definiteness, subjects, topics, and point of view. In: Li, Charles (Hg.): Subject and topic. New York u.a., S. 25-55.

Chomsky, Noam (1995): The Minimalist program. (= Current Studies in Linguistics Series 28). Cambridge, MA u.a.

Chomsky, Noam/Halle, Morris (1968): The sound pattern of English. New York u.a.

Declerck, Renaat (1984): The pragmatics of it-clefts and wh-clefts. In: Lingua 64, S. 251289.

Doherty, Monika (2003): Parametrized beginnings of sentences in English and German. In: Across Languages and Cultures 4, 1, S. 19-51.

Dretske, Fred I. (1972): Contrastive statements. In: The Philosophical Review 81, S. 411-437.

Duden 4 (1998): Duden. Bd. 4: Grammatik. 6., neu bearb. Aufl. Hrsg. v. Wissenschaftlichen Rat der Dudenredaktion. Mannheim/Wien/Zürich.

É.Kiss, Katalin (1998): Identificational vs. information focus. In: Language 74, 2, S. 245-273. 
Fanselow, Gisbert/Lenertová, Denisa (2011): Left peripheral focus: mismatches between syntax and information structure. In: Natural Language and Linguistic Theory 29, 1, S. 169-209.

Frey, Werner (2006): The grammar-pragmatics interface and the German prefield. In: Molnár/Winkler (Hg.), S. 235-264.

Frey, Werner (2010): A-movement and conventional implicatures: about the grammatical encoding of emphasis in German. In: Lingua 120.6, S. 1416-1435.

Fries, Norbert (1988): Über das Null-Topik im Deutschen. In: Sprache und Pragmatik. Arbeitsbericht 3, Lund, S. 19-49

Gécseg, Zsuzsanna/Kiefer, Ferenc (2009): A new look at information structure in Hungarian. In: Natural Language and Linguistic Theory 27, 3, S. 583-622.

Givón, Talmy (1988): The pragmatics of word order: predictability, importance and attention. Studies in syntactic typology. In: Hammond/Moravcsik/Wirth (Hg.), S. 243284.

Gundel, Jeanette (1988): Universals of topic-comment. In: Hammond/Moravcsik/Wirth (Hg.), S. 209-239.

Gundel, Jeanette/Hedberg, Nancy/Zacharski, Ron (1993): Cognitive status and the form of referring expressions. In: Language 69, S. 274-307.

Hammond, Michael/Moravcsik, Edith/Wirth, Jessica R. (Hg.) (1988): Studies in syntactic typology. (= Typological Studies in Language 17). Amsterdam/Philadelphia.

Herring, Susan (1990): Information structure as a consequence of word order type. In: Proceedings of the Sixteenth Annual Meeting of the Berkeley Linguistic Society. February 16-19, 1990. Berkeley, S. 163-174.

Hetland, Jorunn/Molnár, Valéria (Hg.) (2003): Structures of focus and grammatical relations. (= Linguistische Arbeiten 477). Tübingen.

Hetzron, Robert (1975): The presentative movement or why the ideal word order is V.S.O.P. In: Li, Charles N. (Hg.): Word order and word order change. Austin u.a., S. 347-388.

Horvath, Julia (2007): Separating focus movement from focus. In: Karimi, Simin/Samiian, Vida/Wilkins, Wendy K. (Hg.): Phrasal and clausal architecture: syntactic derivation and interpretation. (= Linguistik aktuell 101). Amsterdam u.a., S. 108-145.

Huber, Stefan (2002): Es-Clefts und det-Clefts. Zur Syntax, Semantik und Informationsstruktur von Spaltsätzen im Deutschen und Schwedischen. (= Lunder germanistische Forschungen 64). Stockholm.

Huber, Stefan (2006): The complex functions of it-Clefts. In: Molnár/Winkler, S. 549_ 579.

Jackendoff, Ray (2002): Foundations of language. Brain, meaning, grammar, evolution. Oxford u.a.

Järventausta, Marja (2003): Are Finnish null subjects null topics? In: Hetland/Molnár (Hg.), S. 87-110. 
Krylowa, Olga/Chavronina, Svetlana (1976): Porjadok slov v russkom jazyke [= Die Wortstellung im Russischen]. Moskau.

Lambrecht, Knud (1994): Information structure and sentence form. Topic, focus, and the mental representation of discourse referents. (= Cambridge Studies in Linguistics 71). Cambridge u.a.

Larsson Ringqvist, Eva (2003): Ordföljd och informationsstruktur i franska och svenska. (= Acta Vexionensia 27/23). Växjö.

Mehlhorn, Grit (2002): Kontrastierte Konstituenten im Russischen. Experimentelle Untersuchungen zur Informationsstruktur. (= Europäische Hochschulschriften Reihe 16: Slavische Sprachen und Literaturen 65). Frankfurt a.M. u.a.

Mithun, Marianne (1987): Is basic word order universal? In: Tomlin, Russell S. (Hg.): Coherence and grounding in discourse. Outcome of a symposium, Eugene, Oregon, June 1984. (= Typological Studies in Language 11). Amsterdam u.a., S. 282-299.

Molnár, Valéria (1998): Topic in focus. On the syntax, phonology, semantics and pragmatics of the so-called ,constrastive topic' in Hungarian and German. In: Acta Linguistica Hungarica - An International Journal of Linguistics 45, 1-2, S. 1-77.

Molnár, Valéria (2002): Contrast - from a contrastive perspective. In: Hasselgård, Hilde et al. (Hg.): Information structure in a cross-linguistic perspective. (= Language and Computers 39). Amsterdam/New York, S. 147-162.

Molnár, Valéria (2003): C. In: Delsing, Lars-Olof et al. (Hg.): Grammar in Focus. Festschrift for Christer Platzack 18 november 2003. Bd. II. Lund, S. 235-248.

Molnár, Valéria (2006): On different kinds of contrast. In: Molnár/Winkler (Hg.), S. $197-233$.

Molnár, Valéria/Järventausta, Marja (2003): Discourse configurationality in Finnish and Hungarian. In: Hetland/Molnár (Hg.), S. 231-262.

Molnár, Valéria/Winkler, Susanne (Hg.) (2006): Architecture of focus. (= Studies in Generative Syntax 82). Berlin.

Molnár, Valéria/Winkler, Susanne (2009): Edges and gaps: contrast at the interfaces. In: Repp, Sophie/Cook, Philippa (Hg.): Contrast as an information-structural notion in grammar. Lingua Special Issue, S. 1-24.

Neeleman, Ad/Titov, Elena (2009): Focus, contrast, and stress in Russian. In: Linguistic Inquiry 40, S. 514-524.

Prince, Ellen (1978): A comparison of $w h$-clefts and it-clefts in discourse. In: Language 54, S. 883-906.

Prince, Ellen (1981): Toward a taxonomy of given-new information. In: Cole, Peter (Hg.): Radical Pragmatics. New York u.a., S. 223-255.

Prince, Ellen (1984): Topicalization and left-dislocation: a functional analysis. In: White, Sheila J./Teller, Virginia (Hg.): Discourses in reading and linguistics. (= Annuals of the New York Academy of Sciences 433). New York, S. 213-225.

Prince, Ellen (1985): Fancy syntax and ,shared knowledge‘. In: Journal of Pragmatics 9, S. $65-81$. 
Reinhart, Tanja (1982): Pragmatics and linguistics: an analysis of sentence topics. Reproduced by the Indiana University Linguistics Club. Bloomington, IN.

Rizzi, Luigi (1997): The fine structure of the left periphery. In: Haegeman, Liliane (Hg.): Elements of grammar: A handbook of generative syntax. (= Kluwer International Handbooks of Linguistics 1). Dordrecht u.a., S. 281-337.

SAG = Teleman, Ulf/Hellberg, Staffan/Andersson, Erik (1999): Svenska Akademins Grammatik. 4 Bde. Stockholm.

Sasse, Hans-Jürgen (1987): The thetic/categorical distinction revisited. In: Linguistics 25, S. 511-580.

Speyer, Augustin (2004): Competing constraints on Vorfeldbesetzung in German. In: Shaer, Benjamin/Frey, Werner/Maienborn, Claudia (Hg.): Proceedings of the Dislocated Elements Workshop: ZAS Berlin, November 2003. Bd. 2. (= ZAS Papers in Linguistics 35.2). Berlin, S. 519-541.

Speyer, Augustin (2008): German Vorfeld-filling as constraint interaction. In: Benz, Anton/Kühnlein, Peter (Hg.): Constraints in discourse. (= Pragmatics \& Beyond, New Series 172). Amsterdam/Philadelphia, S. 267-290.

Tomlin, Russell S. (1986): Basic word order. Functional principles. London u.a.

Vallduví, Enric (1992): The informational component. New York/London.

Vallduví, Enric/Engdahl, Elisabeth (1996): The linguistic realization of information packaging. In: Linguistics 34, S. 459-519.

Vallduví, Enric/Vilkuna, Maria (1998): On rheme and kontrast. In: Culicover, Peter W./ McNally, Louise (Hg.): The limits of syntax. (= Syntax and Semantics 29). San Diego u.a., S. 79-108.

Vilkuna, Maria (1995): Discourse configurationality in Finnish. In: É.Kiss, Katalina (Hg.): Discourse configurational languages. New York u.a., S. 244-268.

Zybatow, Gerhild/Junghanns, Uwe (1998): Topiks im Russischen. (= Sprache \& Pragmatik 47). Lund.

Zybatow, Gerhild/Mehlhorn, Grit (2000): Experimental evidence for focus structure in Russian. In: King, T. Holloway/Sekerina, Irina A. (Hg.): Annual workshop on formal approaches to Slavic linguistics. The Philadelphia meeting 1999. (= Michigan Slavic Materials 45). Ann Arbor, S. 414-434. 